\title{
CONSTITUINTES QUÍMICOS E AVALIAÇÃO DO POTENCIAL ANTIINFLAMATÓRIO E ANTIOXIDANTE DE EXTRATOS DAS FOLHAS DE Chomelia obtusa CHAM. \& SCHLTDL. (RUBIACEAE)
}

\author{
Michely Pereira de Barros, Silvana Maria de Oliveira Santin, Willian Ferreira da Costa, Gentil José Vidotti e Maria Helena \\ Sarragiotto* \\ Departamento de Química, Universidade Estadual de Maringá, 87020-900 Maringá - PR, Brasil \\ Maria Conceição de Souza \\ Departamento de Biologia, Universidade Estadual de Maringá, 87020-900 Maringá - PR, Brasil \\ Ciomar Aparecida Bersani-Amado \\ Departamento de Farmácia e Farmacologia, Universidade Estadual de Maringá, 87020-900 Maringá - PR, Brasil
}

Recebido em 28/9/07; aceito em 12/6/08; publicado na web 31/10/08

\begin{abstract}
CHEMICAL CONSTITUENTS AND ANTI-INFLAMMATORY AND ANTIOXIDANT ACTIVITIES EVALUATION OF THE LEAVES EXTRACTS OF Chomelia obtusa CHAM. \& SCHLTDL. (RUBIACEAE). The phytochemical investigation of Chomelia obtusa leaves led to the isolation of four triterpenes (3-O- $\beta$-D-quinovopyranosyl-28-O- $\beta$-D-glycopyranosyl quinovic acid, 3-O- $\beta$-Dquinovopyranosyl-28- $O$ - $\beta$-D-glycopyranosyl cincholic acid, and a mixture of ursolic and oleanolic acids), two flavonoids (3-O- $\beta$-Dglycopyranosyl quercetin, 3-O-[ $\alpha$-L-rhamnopyranosyl-(1 $\rightarrow 6)-\beta$-D-galactopyranoside] quercetin), besides bornesitol and a mixture of 3,5- and 4,5-O-dicaffeoyl quinic acids. The structures of the isolated compounds were assigned on the basis of spectroscopic data, including two-dimensional NMR methods. The anti-inflammatory and antioxidant activities of the crude methanolic extract and of its fractions were evaluated. This is the first report on the chemical and biological investigation of the Chomelia genus.
\end{abstract}

Keywords: Chomelia obtusa; Guettardeae; chemical constituents.

\section{INTRODUÇÃO}

A planície de inundação do alto rio Paraná, abrangendo parte da Área de Proteção Ambiental (APA) das Ilhas e Várzeas do Rio Paraná (Porto Rico-PR e MS), ${ }^{1}$ tem sido alvo de pesquisa de nosso grupo, com o objetivo de avaliar o potencial químico e farmacológico das plantas nativas daquela região. Nossos estudos visam contribuir para a preservação da biodiversidade da referida APA, bem como dar suporte à classificação das espécies vegetais presentes, com base em quimiotaxonomia. Na região, a família Rubiaceae está representada por 22 espécies distribuídas em 18 gêneros e 3 subfamílias, ${ }^{1}$ podendo-se destacar a subfamília Guettardoideae-tribo Guettardeae, com a ocorrência dos gêneros Machaonia, Guettarda e Chomelia. Dentre os poucos estudos químicos encontrados desta tribo constata-se a presença de alcalóides indólicos e quinolínicos, ácidos clorogênicos, triterpenos glicosilados e iridóides como principais constituintes, ${ }^{2-7}$ tendo o gênero Guettard $a^{2}$ o maior número de espécies investigadas.

Em trabalhos anteriores, com a tribo Guettardeae, realizamos os estudos das espécies Machaonia brasiliensis e Guettarda pohliana, os quais revelaram a presença do iridóide secologanosídeo e de flavonóides, ${ }^{6}$ de ácidos clorogênicos, ${ }^{6,7}$ dos ácidos quinóvico, ursólico e oleanóico ${ }^{7}$ e de saponinas derivadas dos ácidos quinóvico e cinchólico. ${ }^{7}$ A presença de secologanosídeo em $M$. brasiliensis foi considerada de importância quimiotaxonômica para estudos da subfamília Guettardoideae e da tribo Guettardeae. ${ }^{6}$

Em continuidade ao nosso projeto de bioprospecção com plantas nativas da planície alagável do alto rio Paraná, especialmente da tribo Guettardeae, neste trabalho relatamos a investigação química e a avaliação das atividades antiinflamatória e antioxidante da espécie Chomelia obtusa Cham. \& Schltdl. A investigação de C. obtusa contribuirá para os

*e-mail: mhsarragiotto@uem.br estudos da tribo Guettardeae, uma vez que este corresponde ao primeiro relato sobre constituintes químicos do gênero Chomelia.

\section{PARTE EXPERIMENTAL}

\section{Procedimentos experimentais gerais}

Os espectros de RMN (uni- e bidimensionais) foram obtidos em espectrômetro Varian, modelo Mercury plus BB, operando a 300 $\mathrm{MHz}$ para ${ }^{1} \mathrm{He}$ e 75,5 MHz para ${ }^{13} \mathrm{C}$. Os deslocamentos químicos foram dados em ppm, tendo como referência interna o tetrametilsilano TMS ( $\delta=0,0 \mathrm{ppm}$ ) ou o próprio solvente. Os solventes utilizados foram $\mathrm{D}_{2} \mathrm{O}, \mathrm{CD}_{3} \mathrm{OD}$ e $\mathrm{CDCl}_{3}$. Para as cromatografias em coluna (CC) foi utilizado gel de sílica 60 (0,063-0,200 mm, Merck) ou Sephadex LH-20, como fase estacionária. A quantidade de adsorvente e as dimensões das colunas variaram de acordo com a quantidade de material empregado. Para as cromatografias em camada delgada (CCD) e em camada delgada preparativa (CCDP) empregou-se gel de sílica $60 \mathrm{G}$ e $60 \mathrm{GF}_{254}$ (Merck), suspenso em água destilada, numa proporção de 1:2, e distribuído em espessura de 0,25 e 1,0 mm, respectivamente. A visualização dos compostos em CCD foi realizada por irradiação com luz ultravioleta em 254 e $366 \mathrm{~nm}$ e/ou por pulverização com solução de $\mathrm{H}_{2} \mathrm{SO}_{4} / \mathrm{MeOH}$ (1:1), $\mathrm{H}_{2} \mathrm{SO}_{4}$ /anisaldeído/ácido acético (1:0,5:50 $\mathrm{mL})$ seguido de aquecimento.

\section{Material vegetal}

A planta Chomelia obtusa (folhas) foi coletada em dezembro de 2001, às margens da planície de inundação do rio Paraná, na região de Porto Rico-PR e identificada pela $\operatorname{Dr}^{\mathrm{a}}$ M. C. de Souza, do Departamento de Biologia da UEM. Uma exsicata do material vegetal encontra-se depositada no Herbário do NUPÉLIA da UEM, sob registro HUEM 13.517. 


\section{Isolamento dos constituintes químicos}

O material vegetal (405,0 g), após seco e moído, foi extraído por maceração em metanol. O solvente foi eliminado sob vácuo em evaporador rotativo fornecendo o extrato metanólico (68,0 g). Parte deste extrato $(37,0 \mathrm{~g})$ foi submetido a um pré-fracionamento em coluna de gel de sílica 60 utilizando-se $\mathrm{CHCl}_{3}, \mathrm{CHCl}_{3} / \mathrm{MeOH}$ 1:1 e $\mathrm{MeOH}$ como eluentes, obtendo-se as frações $\mathrm{CHCl}_{3}$ (COC, 580,0 $\mathrm{mg}$ ), $\mathrm{CHCl}_{3} / \mathrm{MeOH}$ (COCM, 18,5 g) e $\mathrm{MeOH}$ (COM, 14,0 g). A purificação da fração $\mathrm{COC}(370,0 \mathrm{mg})$ por coluna cromatográfica em gel de sílica 60 empregando-se como eluentes misturas de hexano/ AcOEt 9:1, 7:3 e 1:1 e AcOEt forneceu uma mistura constituída pelas substâncias 1 e 2 (4,2 mg). Parte da fração COCM (6,4 g) foi submetida à coluna cromatográfica em gel de sílica 60 e eluída em $\mathrm{CHCl}_{3}, \mathrm{CHCl}_{3} / \mathrm{MeOH}$ 9:1, 8:2, 7:3 e 1:1 e $\mathrm{MeOH}$, obtendo-se 18 subfrações (COCM-1 a COCM-18). A subfração COCM-10 (504,0 $\mathrm{mg}$ ), eluída em $\mathrm{CHCl}_{3} / \mathrm{MeOH}$ 8:2, foi submetida a sucessivas filtrações em coluna de Sephadex LH-20, empregando-se como eluentes $\mathrm{H}_{2} \mathrm{O}, \mathrm{H}_{2} \mathrm{O} / \mathrm{MeOH} 3: 1,1: 1$ e MeOH. Este procedimento resultou no isolamento das substâncias $\mathbf{3}$ (17,8 mg) e 4 (12,2 mg), na forma pura, e na obtenção de diversas subfrações contendo estas substâncias como mistura binária. Purificação da subfração COCM-11 (393,0 $\mathrm{mg}$ ), eluída em $\mathrm{CHCl}_{3} / \mathrm{MeOH}$ 7:3 em coluna de Sephadex LH-20, empregando-se $\mathrm{H}_{2} \mathrm{O}, \mathrm{H}_{2} \mathrm{O} / \mathrm{MeOH}$ 3:1, 1:1, 3:7 e 1:9 e MeOH forneceu as substâncias $5(6,9 \mathrm{mg})$ e $\mathbf{6}(5,0 \mathrm{mg})$, além de uma mistura de 3 e 4 (240,0 mg). O mesmo procedimento foi empregado para a purificação da subfração COCM-18 (320,0 mg), eluída em $\mathrm{CHCl}_{3} /$ $\mathrm{MeOH}$ 1:1, o que resultou no isolamento da substância 7 (16,6 mg) e uma mistura de 8 e $\mathbf{9}$ (43,0 $\mathrm{mg})$.

As frações COCM-1 a COCM-9 apresentaram espectros de $\mathrm{RMN}^{1} \mathrm{H}$ característicos de ácidos graxos e não foram estudadas. Os espectros de $\mathrm{RMN}^{1} \mathrm{H}$ das frações COCM-12 e COCM-14 a COCM-17 mostraram que eram compostas pelas mesmas substâncias já isoladas das frações estudadas.

Parte da fração COM (405,0 mg), obtida do fracionamento do extrato bruto, foi submetida à filtração em Sephadex LH-20 utilizando como eluentes $\mathrm{H}_{2} \mathrm{O}, \mathrm{H}_{2} \mathrm{O} / \mathrm{MeOH}$ 3:1, 1:1, 3:7 e 1:9 e $\mathrm{MeOH}$, o que resultou em 24 subfrações (COM-1 a COM-24). A cromatografia da fração COM-11, em Sephadex LH-20, forneceu uma mistura das substâncias 8 e 9 (40,5 mg). Os espectros de $\mathrm{RMN}^{1} \mathrm{H}$ das demais subfrações mostraram a presença de misturas dos flavonóides 5 e 6 e dos ácidos clorogênicos $\mathbf{8}$ e $\mathbf{9}$, além de açúcares livres.

\section{Ensaios biológicos}

\section{Avaliação da atividade antiinflamatória}

Empregou-se o modelo de edema de orelha induzido por óleo de cróton em camundongo. ${ }^{8}$ Foram utilizados camundongos Swiss machos e fêmeas, pesando 25-30 g, em média. O óleo de cróton foi diluído em uma solução de acetona/água 7:3 e aplicado, na concentração de $200 \mu \mathrm{g} /$ orelha, em ambas as faces internas das orelhas dos camundongos. Uma solução ( $20 \mu \mathrm{L})$ do extrato bruto metanólico e da fração COCM foi aplicada na superfície interna da orelha esquerda (2,5 mg/orelha). A orelha direita recebeu apenas o solvente utilizado para solubilização das amostras $(20 \mu \mathrm{L})$. Após $6 \mathrm{~h}$, os animais foram sacrificados e as orelhas seccionadas em discos circulares de $6 \mathrm{~mm}$ de diâmetro. Estas secções foram pesadas em uma balança analítica. A indometacina foi usada como controle positivo. Os resultados foram expressos como média \pm erro padrão da média (epm) e analisados utilizando teste de Student para comparação de duas médias, ou análise de variância (ANOVA) para múltiplas comparações, sendo utilizado $\mathrm{P}<0,05$ como nível de significância.

\section{Avaliação da atividade antioxidante}

Os testes de atividade antioxidante foram realizados para o extrato bruto metanólico, frações COCM e COM e subfrações COCM-10, COCM-11 e COCM-18. Os potenciais de atividade antioxidante foram determinados com base na atividade seqüestradora de radical livre do 2,2-difenil-1-picril-hidrazil (DPPH). ${ }^{9}$ As amostras foram adicionadas em diferentes concentrações a $2 \mathrm{~mL}$ de uma solução de DPPH (4,7 mg em 75 mL de metanol). Foram empregadas 10 diferentes concentrações, na faixa de 0 a $140 \mu \mathrm{g} \mathrm{mL}^{-1}$ para COCM-10 e de 0 a $73 \mu \mathrm{g} \mathrm{mL}^{-1}$, para as demais amostras. Após 30 min a absorbância foi determinada em espectrofotômetro UV-visível, a $515 \mathrm{~nm}$, empregando metanol como branco. Os testes foram realizados em triplicata. Uma solução de DPPH sem adição das amostras foi utilizada como controle. O BHT foi utilizado como padrão. A capacidade seqüestradora de radicais livres foi determinada utilizando a análise de regressão linear no intervalo de confiança de $95 \%(\mathrm{P}<0,05)$. Os resultados foram expressos como $\mathrm{IC}_{50}$, que corresponde à concentração da amostra necessária para sequiestrar $50 \%$ de radicais livres.

\section{RESULTADOS E DISCUSSÃO}

Inicialmente, extratos do material vegetal foram submetidos a ensaios qualitativos para a detecção de alcalóides e/ou iridóides, ${ }^{10}$ os quais representam constituintes comumente encontrados na família Rubiaceae. Os resultados dos ensaios revelaram a ausência destas substâncias nos extratos das folhas de Chomelia obtusa.

O extrato bruto metanólico foi então fracionado em coluna cromatográfica em gel de sílica, fornecendo as frações clorofórmio (COC), clorofórmio/metanol (COCM) e metanol (COM). A purificação das frações obtidas, por sucessivas cromatografias em gel de sílica ou Sephadex LH-20, resultou no isolamento dos triterpenos 1-4, dos flavonóides glicosilados $\mathbf{5}$ e $\mathbf{6}$, do açúcar $\mathbf{7}$ e de uma mistura dos ácidos clorogênicos 8 e 9.

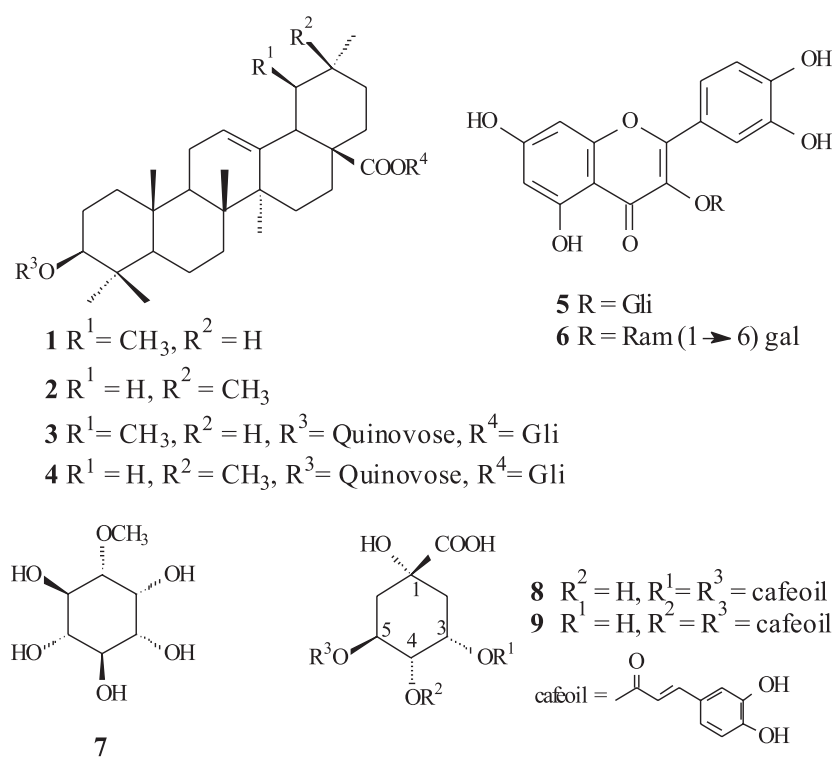

A análise dos espectros 1D e 2D de $\mathrm{RMN}{ }^{1} \mathrm{He} \mathrm{e}^{13} \mathrm{C}$ e comparação com dados descritos na literatura permitiu a identificação das estruturas das substâncias 1-6 como sendo ácido ursólico (1), ${ }^{11}$ ácido oleanólico (2), ${ }^{11}$ ácido 3-O- $\beta$-D-quinovopiranosil-28-O- $\beta$-D-glucopiranosil quinóvico (3), ${ }^{12}$ ácido 3- $O$ - $\beta$-D-quinovopiranosil-28- $O$ - $\beta$-D-glucopiranosil cinchólico (4), ${ }^{12} 3-O-\beta$-D-glucopiranosil quercetina $(5)^{13}$ e $3-O$-[ $\alpha$-raminosil$(1 \rightarrow 6)$ - $\beta$-galactopiranosideo] quercetina $(6) .{ }^{13} \mathrm{~A}$ definição da posição das unidades glicosídicas para as substâncias 3-6 foi realizada com base nas correlações observadas nos respectivos espectros de HMBC. Além 
disto, no caso das substâncias 3 e 4, o valor do deslocamento químico do $\mathrm{C}-3\left(\delta_{\mathrm{C}} 90,7\right)$ corrobora a presença de uma unidade glicosídica neste carbono, uma vez que este sinal aparece em $\delta_{\mathrm{C}} 77,0(\mathrm{C}-3)$ para os ácidos quinóvico e cinchólico não glicosilados nesta posição. ${ }^{11}$

A comparação dos dados espectroscópicos de $\mathrm{RMN}{ }^{1} \mathrm{H}$ e ${ }^{13} \mathrm{C}$ com os da literatura permitiu a identificação da substância 7 como 1- $O$-metilinositol (bornesitol). ${ }^{14} \mathrm{O}$ estabelecimento da estereoquímica relativa de 7 foi baseado nos valores das constantes de acoplamento e nas correlações entre os hidrogênios observadas no espectro de COSY.

As substâncias 8 e 9 foram caracterizadas como ácidos 3,5- e 4,5-O-dicafeoilquínico, respectivamente, através da comparação dos dados espectroscópicos de $\mathrm{RMN}{ }^{1} \mathrm{H}$ e ${ }^{13} \mathrm{C}$ com os descritos na literatura para os possíveis isômeros do ácido dicafeoilquínico. ${ }^{6,15} \mathrm{~A}$ presença de duas unidades cafeoíla foi evidenciada, principalmente, pelos sinais de dois grupos de hidrogênios olefínicos trans na região de $\delta_{\mathrm{H}} 7,40$ (d, J 16 Hz, H-7'/H-7'”) e 6,00 (d, J 16 Hz, H-8'/H-8'), além dos sinais para hidrogênios e carbonos dos sistemas aromáticos. As posições dos grupos cafeoíla foram determinadas com base nos valores de deslocamentos químicos para H-3, H-4 e H-5 e comparação destes com os dos possíveis isômeros resultantes da substituição de duas hidroxilas do ácido quínico por unidades cafeoíla. ${ }^{6,15}$

A ocorrência de derivados glicosilados do ácido quinóvico e de ácidos clorogênicos já foi relatada anteriormente na tribo Guettardeae. Das espécies Timonius timon, ${ }^{4}$ Antirhea chinensis ${ }^{5}$ e de algumas do gênero Guettard $a^{2}$ foram isolados derivados do ácido quinóvico contendo unidades glicosídicas ligadas ao C-3 e C-28, como no caso da substância 3. A presença da aglicona foi detectada em Guettarda e na espécie Antirhea chinensis. ${ }^{5}$ Ácidos quínicos contendo a unidade cafeoíla, como em $\mathbf{8}$ e 9, foram isolados de Guettarda ${ }^{2}$ e de Machaonia brasiliensis. ${ }^{6}$

O isolamento dos triterpenos 3 e 4 e dos ácidos quínicos 8 e 9, bem como a ausência de secoiridóides e alcalóides indólicos em Chomelia obtusa, verificada através dos testes qualitativos realizados e pelos espectros de RMN ${ }^{1} \mathrm{H}$ obtidos para todas as subfrações, representam dados importantes para futuros estudos quimiotaxonômicos envolvendo o gênero Chomelia e a tribo Guettardeae.

$\mathrm{O}$ extrato bruto e algumas das frações e subfrações resultantes do fracionamento deste foram submetidas a ensaios biológicos para avaliação de suas atividades antiinflamatória e antioxidante.

A atividade antiinflamatória foi determinada para o extrato bruto e para a fração COCM, utilizando-se o modelo de edema de orelha induzido por óleo de cróton em camundongo. Os resultados mostraram que a administração do extrato bruto e da fração COCM, na dose de 2,5 mg por via tópica, produziram 24 e $57 \%$ de inibição do edema de orelha, respectivamente.

$\mathrm{Na}$ avaliação da atividade antioxidante foi empregado o método do DPPH, metodologia muito utilizada para medir a capacidade seqüestradora de radicais livres de produtos naturais. ${ }^{9}$ Os resultados deste ensaio (Tabela 1) mostraram que o extrato bruto e a fração COM apresentaram significativa atividade seqüestradora de radicais livres, com $\mathrm{IC}_{50}$ compa-

Tabela 1. Valores de $\mathrm{IC}_{50}(\mu \mathrm{g} / \mathrm{mL})$ para extrato bruto, frações e subfrações obtidas de Chomelia obtusa

\begin{tabular}{lc}
\hline Amostra & $\mathrm{IC}_{50}($ intervalo de confiança de $95 \%)$ \\
\hline Extrato bruto & $15,19(14,49-15,98)$ \\
COCM & $27,86(26,58-29,17)$ \\
COM & $13,76(12,49-15,02)$ \\
COCM-10 & $82,45(76,52-89,10)$ \\
COCM-11 & $52,88(47,14-58,74)$ \\
COCM-18 & $22,85(22,02-23,80)$ \\
BHT & $16,90(14,30-20,10)$ \\
\hline
\end{tabular}

ráveis ao do BHT, antioxidante comercial utilizado como padrão. Como constatado na parte experimental, a fração COM é constituída basicamente pelos flavonóides 5 e 6 e pelos ácidos clorogênicos 8 e 9, sendo estes, provavelmente, os responsáveis pela atividade antioxidante apresentada. Trabalhos descritos na literatura têm demonstrado que os ácidos 3,5- e 4,5-O-dicafeoilquínico (8) e $(\mathbf{9})^{16}$ e derivados 3-O-glicosilados da quercetina ${ }^{17}$ apresentam significativas capacidades seqüestradoras de radicais livres, com valores de $\mathrm{IC}_{50}$ próximos aos obtidos para a fração COM.

\section{AGRADECIMENTOS}

À Fundação Araucária-PR, pelo apoio financeiro.

\section{REFERÊNCIAS}

1. Souza, M. C.; Cislinski, J.; Romagnolo, M. B. Em A planície de Inundação do Alto Rio Paraná: Aspectos Físicos, Químicos e Socioeconômicos; Vazzoler, A. E. A. M.; Agostinho, A. A.; Hahn, N. S., eds.; Eduem: Maringá, 1997, cap. II.12.

2. Sousa, M. P.; Matos, M. E. O.; Machado, M. I. L.; Vencato, I.; Mascarenhas, Y. P.; Braz Filho, R.; Phytochemistry 1984, 23, 2589; Bhattacharyya, J.; Almeida, M. Z.; J. Nat. Prod. 1985, 48, 148; KanFan, C.; Brillanceau, M. H.; Husson, H. P.; J. Nat. Prod. 1986, 49, 1130; Sousa, M. P.; Matos, M. E. O.; Machado, M. I. L.; Braz Filho, R.; Phytochemistry 1986, 25, 1419; Montagnac, A.; Litaudon, M.; Pais, M.; Phytochemistry 1987, 46, 973; Aquino, R.; Simone, F.; Pizza, C.; Cerri, R.; Mello, J. F.; Phytochemistry 1988, 27, 2927; Senatore, F.; Aquino, R.; Simone, F.; Pizza, C.; Pharm. Res. Comm. 1988, 20, 105; Aquino, R.; De Simone, F.; Pizza, C.; Mello, J.; Phytochemistry 1989, 29, 199; Capasso, A.; Balderrama, L.; Sivila, S. C.; Tommasi, N. D.; Sorrentino, L.; Pizza, C.; Planta Med. 1998, 64, 348.

3. Weniger, B.; Jiang, Y.; Anton, R.; Bastida, J.; Varea, T.; Quirion, J.-C.; Phytochemistry 1993, 32, 1587.

4. Khan, I. A.; Rali, T.; Sticher, O.; J. Nat. Prod. 1993, 56, 2163.

5. Hui, W. H.; Szeto, S. K.; Phytochemistry 1967, 6, 443.

6. Santos, A. R.; Barros, M. P.; Santin, S. M. O.; Souza, M. C.; Eberlin, M. N.; Meurer, E. C.; Sarragiotto, M. H.; Quim. Nova 2004, 27, 525.

7. Oliveira, P. R. N.; Testa, G., Sena, S. B.; Sarragiotto, M. H.; Costa, W. F.; Souza, M. C.; Santin, S. M. O.; Quim. Nova 2008, 31, 755.

8. Van Arman, G. C.; Clin. Pharmacol. Ther. 1974, 16, 900.

9. Silva, F. A. M.; Borges, M. F. M.; Ferreira, M. A.; Quim. Nova 1999, 22, 24; Cho, E. J.; Yokozawa, T.; Rhyu, D. Y.; Kim, S. C.; Shibahara, N.; Park, J. C.; Phytomedicine 2003, 10, 544.

10. Harborne, J. B.; Phytochemical Methods: A Guide to Modern Techniques of Plant Analysis, Chapman and Hall: London, 1973.

11. Mahato, S. B.; Kundu, A. P.; Phytochemistry 1994, 37, 1517.

12. Arriaga, F. J.; Rumbero, E.; Vazquez, P.; Phytochemistry 1990, 29, 209.

13. Agrawal, P. K.; Carbon-13 NMR of flavonoids, Elsevier: Netherlands, 1989; vol. 39 .

14. Ichimura, K.; Kohata, K.; Mukasa, Y.; Yamaguchi, Y.; Goto, R.; Suto, K., Biosci. Biotechnol. Biochem. 1999, 63, 189.

15. Cheminat, A.; Zawatzky, R.; Becker, H.; Brouillard, R.; Phytochemistry 1988, 27, 2787; Kwon, H. C.; Jung, C. M.; Shin, C. G.; Lee, J. K.; Choi, S. U.; Kim, S. Y.; Lee, K. R.; Chem. Pharm. Bull. 2000, 48, 1796.

16. Hung, T. M.; Na, M.; Thuong, P. T.; Su, N. D., Sok, D.; Song, K. S.; Seong, Y. H.; Bae, K.; J. Ethnopharmacol. 2006, 108, 188.

17. Seyoum, A.; Asres. K.; El-Fiky, F. K.; Phytochemistry 2006, 67, 2058. 